\title{
Closed Incision Negative Pressure Therapy for Management of Incision Wounds in the Groin After Revision Vascular Surgery: A Randomized Controlled Trial
}

\author{
Sebastian Paul Pleger ${ }^{1,}$, Liesa Fuhrmann ${ }^{1}$, Mouiad Al Tattan ${ }^{1}$, Alexander Kunold ${ }^{1}$, \\ Meshal Elzien ${ }^{1}$, Andreas Böning ${ }^{2}$, Ahmed Koshty ${ }^{1}$ \\ ${ }^{1}$ Department of Vascular Surgery, Jung-Stilling Hospital, Siegen, Germany \\ ${ }^{2}$ Department of Cardiac and Vascular Surgery, University Hospital, Giessen, Germany
}

Email address:

sebastian.pleger@diakonie-sw.de (S. P. Pleger)

${ }^{*}$ Corresponding author

\section{To cite this article:}

Sebastian Paul Pleger, Liesa Fuhrmann, Mouiad Al Tattan, Alexander Kunold, Meshal Elzien, Andreas Böning, Ahmed Koshty. Closed Incision Negative Pressure Therapy for Management of Incision Wounds in the Groin After Revision Vascular Surgery: A Randomized Controlled Trial. Journal of Surgery. Vol. 9, No. 1, 2021, pp. 36-44. doi: 10.11648/j.js.20210901.17

Received: January 29, 2021; Accepted: February 6, 2021; Published: February 27, 2021

\begin{abstract}
Wound healing complications (WHCs) in the groin after vascular surgeries are a serious problem for patients and surgeons in various surgical disciplines. The incidence of WHCs of up to $44 \%$ after incisions in the groin is often responsible for prolonged hospital stay and high treatment costs. An effective reduction of WHCs for various wound types after using closed incision negative pressure therapy (ciNPT) has been documented in many case reports and clinical studies. As the majority of studies have addressed the effect of ciNPT on primary groin incision wounds, concerning groin incision wounds after revision vascular surgery are extremely scarce. The aim of this prospective, randomized clinical study was to investigate the effectiveness of ciNPT compared with conventional therapy on groin incisions after revision vascular surgery. We analyzed the cases of a total of 94 patients with 100 groin incisions. Patients were randomized and treated with either PREVENA ${ }^{\mathrm{TM}}$ $\left(\mathrm{n}=47\right.$ groins) or a conventional adhesive dressing ( $\mathrm{n}=53$ groins; control group). PREVENA ${ }^{\mathrm{TM}}$ was applied intraoperatively and was removed on day 5,6 or 7 postoperatively. Wound evaluation was carried out on the 5 th to 7 th and 30th postoperative day. Compared with the control group, the ciNPT group showed a reduction in the overall incidence of WHCs assessed 30 days postoperatively $(\mathrm{p}<0.0005)$. With regard to prevention of revision surgeries, the ciNPT had no significant impact $(\mathrm{p}=0.056)$. Subgroup analysis revealed a significant effect of ciNPT for almost all wound healing risk factors. Based on our results, ciNPT provides a promising therapeutic option to reduce the frequency of postoperative WHCs and the need for revision surgeries in the groin after revision vascular surgery in patients with wound healing risk factors.
\end{abstract}

Keywords: Closed Incision Negative Pressure Therapy, Surgical Site Infections, Postoperative Wound Complications, Wound Healing

\section{Introduction}

For decades postoperative wound healing complications (WHCs) have presented serious problems for patients and surgeons in various surgical disciplines. In particular, surgical site infections (SSIs) are an important cause of prolonged hospital stay, unplanned readmissions after surgery, morbidity, and death [1]. With an estimated 157,500 SSIs in the United States per year, this type of healthcare-associated infection creates an increased burden for the health care system [2]. The costs of SSIs are estimated to be US\$ 3.3 billion annually, and are associated with nearly US\$ 1 million additional inpatient-days [3, 4]. Due to its anatomical structures and its function as a leading access for the majority of vascular surgeries and interventions, the groin shows a propensity for postoperative WHCs. Vascular surgery patients have an incidence of WHCs of up to $44 \%$ after incisions in the groin [5-9]. Hematoma, seroma, lymphatic 
leaks, wound infection, and skin necrosis present the main WHCs $[5,6,10]$. In addition to the application of diverse surgical techniques and systemic antibiotic therapy, the treatment of a broad spectrum of WHCs has been enhanced by negative pressure wound therapy (NPWT), which has been proven to be effective in a wide range of wounds [1115]. In recent years a new form of therapy, known as closed incision negative pressure therapy (ciNPT), has resulted in a decreasing rate of SSIs in various incision wounds [5-10, 1624]. The two leading ciNPT systems responsible for these significant effects are PREVENA ${ }^{\mathrm{TM}}$ Incision Management Therapy System (KCI, an ACELITY Company, San Antonio, Texas, USA) and PICO ${ }^{\text {TM }}$ Single Use Negative Pressure Wound Therapy System (Smith \& Nephew, London, UK). Apart from the different amounts of negative pressure employed (PREVENA ${ }^{\mathrm{TM}}-125 \mathrm{mmHg}$; PICO $^{\mathrm{TM}}-80 \mathrm{mmHg}$ ), both ciNPT systems have the same mode of action, which decreases the lateral tension around the incision wound, strengthens the cohesiveness of the edges, enhances oxygen saturation and blood microcirculation within the incision area, removes fluids and infectious materials from the wound, and secures the incision wound from external contamination $[5,6$, 24]. With few exceptions [25-27], study data present a significant reduction of SSIs and other WHCs for groin wounds after vascular surgery using PREVENA ${ }^{\mathrm{TM}}$ and PICO $^{\text {TM }}[5-10,28,29]$. Almost all data in the literature refer to primary groin wounds after vascular procedures, without giving information about the effect of ciNPT on groin incision wounds after revision vascular surgery. Thus far, there is little randomized clinical trial (RCT) data concerning the issue of revision wounds. Gombert et al. published significant data regarding the effectiveness of ciNPT on incision groin wound healing after revision vascular surgery [7], and Lee et al. reported results from patients after vascular surgery with previous cutdown in the groin that did not show a significant effect [27]. Due to the lack of reliable data, we initiated the present RCT to investigate the effectiveness of PREVENA $^{\mathrm{TM}}$ on incision groin wounds after revision vascular surgery and compare in with that of conventional wound dressing. In particular, we examined the incidence of groin WHCs on postoperative days 5-7 and 30 and the incidence of surgery revisions on postoperative day 30 . In addition, to evaluate the effectiveness of PREVENA ${ }^{\mathrm{TM}}$ on patients at risk, we carried out a subgroup analysis of wound healing risk factors and perioperative risk factors.

\section{Materials and Methods}

This prospective, randomized clinical study was approved by the ethics committee of the Münster Medical Chamber and the Wilhelms University of Münster, Münster, Germany. The study was conducted and fully funded by our own department, without any financial or scientific support from KCI (an ACELITY Company, San Antonio, Texas, USA). From 3 August 2017 to 5 November 2018, 94 patients with 100 groin incisions were evaluated. Inclusion criteria were vascular procedures with access at the common femoral artery with at least a 5 $\mathrm{cm}$ longitudinal incision in the groin and at least two of the following wound healing risk factors: previous vascular surgery with a longitudinal groin incision, overweight, diabetes mellitus, age $>60$ years, renal insufficiency, chronic obstructive pulmonary disease (COPD), and hypoalbuminemia. After randomization based on the optimum-biased coin design by Atkinson, the patients were assigned to either the ciNPT group (PREVENA ${ }^{\mathrm{TM}}$ ) or the control group (conventional wound dressing). The preoperative preparation of all patients included a hair shave and sterile skin disinfection with the antiseptic ECOLAB Skinsept ${ }^{\circledR} \quad G \quad$ [Ecolab GmbH Monheim am Rhein, Germany] in the groin area and a perioperative single-dose antibiotic treatment with cefazolin $2 \mathrm{~g}$ (HEXAL ${ }^{\circledR}$ AG Holzkirchen, Germany; administrated intravenously). Before reapproximation of the subcutaneous tissue with Vicryl ${ }^{\mathrm{TM}}$ 3-0 sutures Ethicon ${ }^{\circledR}$ and the skin with Ethilon ${ }^{\mathrm{TM} I I}$ 2-0 sutures Ethicon ${ }^{\circledR}$ (Johnson \& Johnson Medical GmbH, Norderstedt, Germany), a subfascial drain was placed in all patients. Immediately after closure of the incision wound, either a conventional adhesive dressing Medipore ${ }^{\mathrm{TM}}+$ Pad, (3M Poland Manufacturing Sp. z o. o. 51-416 Wrocław, Poland) or PREVENA ${ }^{\mathrm{TM}}$ was applied. The components of PREVENA ${ }^{\mathrm{TM}}$ are a vacuum unit with a battery with a preset negative pressure of $-125 \mathrm{mmHg}$, a replaceable exudate collection canister (volume $45 \mathrm{ml}$ ), a polyester fabric interface layer with $0.019 \%$ silver for the control of bioburden within the dressing, a polyurethane foam bolster, and a polyurethane film with acrylic adhesive. PREVENA ${ }^{\mathrm{TM}}$ was removed on postoperative day 5-7 with subsequent use of the conventional adhesive dressing. The conventional adhesive dressing in the control group was changed daily. In both groups the conventional adhesive dressing was left until suture removal. The first evaluation took place on postoperative day 5-7 during the hospital stay and the second evaluation on postoperative day 30 in the outpatient clinic (Figures 1 and 2). The assessment of the incision wounds was based on a modified Szilagyi classification [30]: grade I superficial infections on the skin surface; grade II infiltration of the subcutaneous layer; grade III infection of the arterial graft. We additionally incorporated different types of postoperative WHCs of our study into this classification system. Thus, cutaneous wound dehiscence, skin necrosis, or isolated local signs of infection were classified as grade I; wound dehiscence in the subcutaneous layer, lymphatic fistula, lymphocele, seroma, hematoma, isolated local signs of infection, or systemic infection parameters (leukocytes $>13 \times 10^{9} / \mathrm{dL}$, C-reactive protein $>100 \mathrm{mg} / \mathrm{L}$ ) were classified as grade II; and arterial graft infections were classified as grade III. Subgroup analysis included all risk factors of wound healing and perioperative risk factors. All risk factors were examined with regard to the incidence of groin WHCs on postoperative days 5-7 and 30 and the need for 
surgical revision until postoperative day 30. The primary risk factors were defined as follows: diabetes mellitus with hemoglobin Alc $(\mathrm{HbAlc})>6.5 \%$ and $48 \mathrm{mmol} / \mathrm{mol}$ glucose; renal insufficiency with glomerular filtration rate $<89 \mathrm{~mL} / \mathrm{min}$ (stage 2) and creatinine > $1.2 \mathrm{mg} / \mathrm{dL}$; overweight with BMI $>25 \mathrm{~kg} / \mathrm{m}^{2}$; hypoalbuminemia with albumin $<3.4 \mathrm{~g} / \mathrm{dL}$; COPD with the Global Initiative For Chronic Obstructive Lung Disease (GOLD) grade $1 \mathrm{FEV}_{1}$ $\geq 80 \%$, and age $>60$ years. Perioperative risk factors were defined as wound length $>10 \mathrm{~cm}$, hospital stay $>18$ days, operative time $>168 \mathrm{~min}$, perioperative blood transfusion with hemoglobin $<8 \mathrm{mg} / \mathrm{dL}$, and previous vascular interventions (digital subtraction angiography or percutaneous transluminal angioplasty). Statistical analysis was performed using Student's test, Levene's test, and Fisher's exact test. Fisher's exact test and the Pearson Chi Square test were used for subgroup analyses. Statistical significance was determined by a p-value $<0.05$.

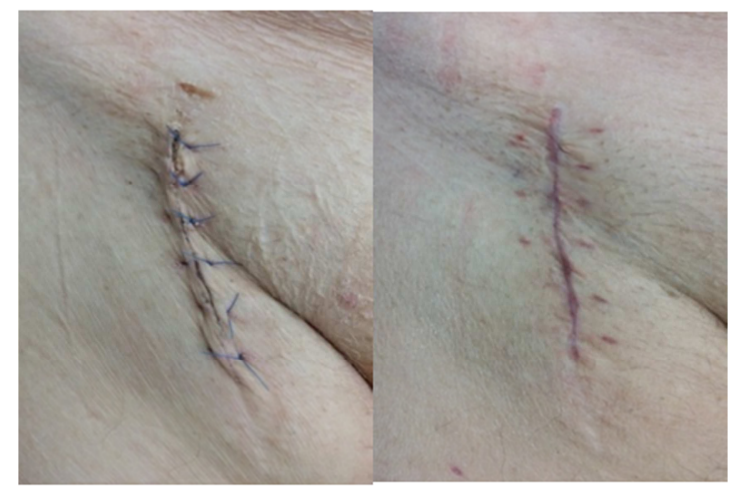

A)

B)

Figure 1. Regular healing process of incision wound in the groin on postoperative days 7 (A) and $30(B)$ in a patient after application of ciNPT.

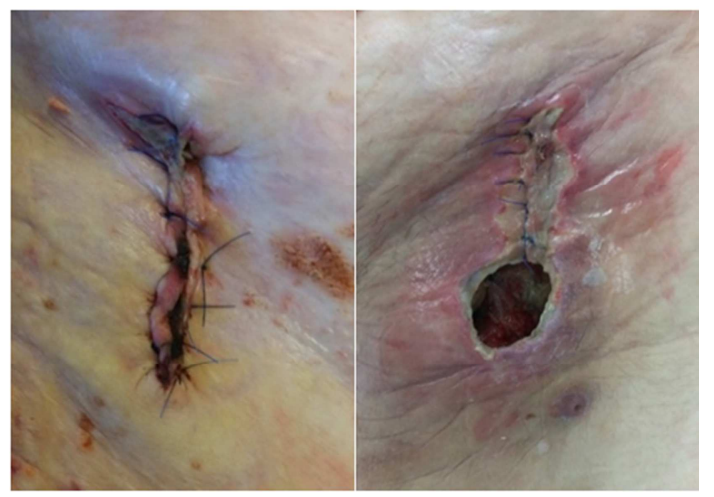

A)

B)

Figure 2. Postoperative wound healling complications. (A) Skin necrosis (day 7) and (B) wound dehiscence in the subcutaneous layer with fat necrosis and isolated local signs of infection (day 14).

\section{Results}

The study included 94 patients with 100 groin wounds. The patient cohort consisted of 25 females and 69 males with a median age of 68.9. Eighty-six groin wounds resulted from unilateral surgeries and 14 were groin wounds from bilateral surgeries such as endovascular aneurysm repair, fenestrated endovascular aneurysm repair, and aortobifemoral bypass. The most frequently reported comorbidity was peripheral artery disease $(86.2 \%)$ (Table 1$)$. The most frequent type of surgery was revascularization $(91.5 \%)$, with the leading procedure being femoral popliteal bypass $(42.5 \%)$ (Table 2$)$. There were a total of 45 groin WHCs $(45 \%)$, including 6 $(12.8 \%)$ in the ciNPT group and $39(73.6 \%)$ in the control group (Table 3). At the first postoperative wound examination on postoperative days 5-7, there were $1(2.1 \%)$ WHC in Szilagyi grade II in the ciNPT group, whereas there were $7(13.2 \%)$ in Szilagyi grade I and 19 (35.8\%) in Szilagyi grade II in the control group. The second postoperative examination on postoperative day 30 showed 1 $(2.1 \%)$ WHC in Szilagyi grade I and 4 (8.5\%) in Szilagyi grade II in the ciNPT group. In the control group there were 4 (7.5\%) WHCs in Szilagyi grade I, 8 (15.1\%) in Szilagyi grade II, and $1(1.9 \%)$ in Szilagyi grade III (Table 3). The difference in incidence on postoperative day 5-7 was statistically significant $(\mathrm{p}<0.0005)$, although this was not the case for postoperative day $30 \quad(\mathrm{p}=0.116)$. The overall incidence of postoperative WHCs assessed 30 days postoperatively showed a more favorable effect of ciNPT over the conventional dressing $(\mathrm{p}<0.0005$; Table 3$)$. There were $2(4.3 \%)$ revision surgeries in the ciNPT group and 9 $(17 \%)$ in the control group; thus, despite the aparrent advantage of ciNPT application of short-term wound healing, no statistical significance $(p=0.056)$ was noted regarding revision surgery (Table 4). The most frequently occurring WHC in the ciNPT group was hematoma (4.2\%), and the leading WHCs in the control group were hematoma (16.9\%), local infection (11.3\%), and lymphatic fistula (11.3\%) (Table 5). Comparison of the two groups showed an advantage of ciNPT in patients with lymphatic fistula $(p=0.028)$. Subgroup analysis of the wound healing risk factors and perioperative risk factors revealed significantly fewer WHCs in the ciNPT group than in the control group for age $(p<0.0005)$, overweight $(p<0.0005)$, diabetes mellitus $(p<0.0005)$, renal insufficiency $(p=0.007)$, hypoproteinemia $(p=0.003)$, wound length $(p<0.0005)$, operation time $(p<0.0005)$, hospital stay $(\mathrm{p}=0.006)$ and perioperative blood transfusion $(\mathrm{p}=0.003)$ (Table 6). On postoperative day 5-7 all risk factors showed an advantage of ciNPT. On postoperative day 30 a beneficial effect of ciNPT was noted only for hospital stay $(p=0.017)$ and wound length $(p=0.026)$. In ciNPT patients with revision surgery, fewer WHCs were observed for the perioperative risk factors wound length $(\mathrm{p}=0.027)$ and hospital stay $(\mathrm{p}=0.017)$ (Table 7). 
Table 1. Patient characteristics.

\begin{tabular}{|c|c|c|c|}
\hline & ciNPT group & Control group & p-value \\
\hline Number of patients & 47 & 47 & \\
\hline Number of groin incisions & 47 & 53 & \\
\hline \multicolumn{4}{|l|}{ Gender } \\
\hline Female & $11(23.4 \%)$ & $14(29.8 \%)$ & 0.641 \\
\hline Male & $36(76.6 \%)$ & $33(70.2 \%)$ & 0.641 \\
\hline Mean age [years] & 71.5 (range 59-84) & 66.8 (range 48-89) & 0.007 \\
\hline Mean BMI $\left[\mathrm{kg} / \mathrm{m}^{2}\right]$ & 27.8 (range 17.8-39.8) & 27.6 (range 18.2-41.9) & 0.881 \\
\hline Hypertension & $41(87.2 \%)$ & $41(87.2 \%)$ & 1 \\
\hline Coronary artery disease & $12(25.5 \%)$ & $18(38.3 \%)$ & 0.268 \\
\hline Diabetes mellitus & $13(27.7 \%)$ & $13(27.7 \%)$ & 1 \\
\hline Renal insufficiency & $19(40.4 \%)$ & $12(25.5 \%)$ & 0.188 \\
\hline Dialysis & $1(2.1 \%)$ & $1(2.1 \%)$ & 1 \\
\hline Hypoproteinemia & $8(17 \%)$ & $24(51.1 \%)$ & 0.001 \\
\hline COPD & $10(21.3 \%)$ & $5(10.6 \%)$ & 0.260 \\
\hline Smoker & $12(25.5 \%)$ & $14(29.8 \%)$ & 0.818 \\
\hline Preoperative anemia & $2(4.3 \%)$ & $6(12.8 \%)$ & 0.267 \\
\hline Postoperative anemia & $31(66 \%)$ & $22(46.8 \%)$ & 0.096 \\
\hline Postoperative leukocytosis & $38(80.9 \%)$ & $21(44.7 \%)$ & 0.001 \\
\hline \multicolumn{4}{|l|}{ Peripheral artery disease } \\
\hline Fontaine classification grade II & $20(42.6 \%)$ & $25(53.2 \%)$ & 0.409 \\
\hline Fontaine classification grade III & $13(27.7 \%)$ & $11(23.4 \%)$ & 0.813 \\
\hline Fontaine classification grade IV & $9(19.1 \%)$ & $3(6.4 \%)$ & 0.120 \\
\hline Infrarenal abdominal aortic aneurysm & $3(6.9 \%)$ & $3(6.9 \%)$ & 1 \\
\hline Thoracic aortic aneurysm & $0(0 \%)$ & $2(4.3 \%)$ & 0.495 \\
\hline Artery occlusion (thrombosis/embolism) & $1(2.1 \%)$ & $3(6.4 \%)$ & 0.617 \\
\hline Iliac artery aneurysm & $0(0 \%)$ & $1(2.1 \%)$ & 1 \\
\hline Leriche syndrome & $1(2.1 \%)$ & $0(0 \%)$ & 1 \\
\hline
\end{tabular}

$\mathrm{BMI}=$ body mass index $\mathrm{COPD}=$ chronic obstructive pulmonary disease

Table 2. Perioperative Characteristics.

\begin{tabular}{llll}
\hline & ciNPT group & Control group & p-value \\
\hline Mean operative time [minutes] & 163.9 (range 37-288) & 172.7 (range 59-380) & 0.497 \\
Mean hospital stay [days] & $16.3($ range 6-45) & 20.9 (range 5-150) & 0.249 \\
Mean wound length [cm] & $9.4($ range 6-14) & 10 (range 5-20) & 0.343 \\
Perioperative blood transfusion & $17(36.2 \%)$ & $15(31.9 \%)$ & 0.828 \\
Procedure types & & & 0.714 \\
EVAR/TEVAR & $3(6.4 \%)$ & $5(10.6 \%)$ & 0.714 \\
Revascularisation & $44(93.6 \%)$ & $42(89.4 \%)$ & 0.773 \\
Bilateral procedures & $6(12.8 \%)$ & $8(17 \%)$ & 0.337 \\
Prosthetic material used & & & 0.052 \\
PTFE & $14(29.8 \%)$ & $9(19.1 \%)$ & 0.089 \\
Dacron & $4(8.5 \%)$ & $12(25.5 \%)$ & 0.223 \\
Biological patch & $11(23.4 \%)$ & $4(8.5 \%)$ & 1 \\
Vein & $14(29.8 \%)$ & $8(17 \%)$ & $2(4.3 \%)$ \\
Composite & $2(4.3 \%)$ & & \\
\hline
\end{tabular}

EVAR=endovascular aortic repair; TEVAR=thoracic endovascular aortic repair; PTFE= Polytetrafluoroethylene, Composite $=$ composite graft of PTFE and saphenous vein

Table 3. Incidence of wound healing disturbances with reference to the total number of groin incisions, wound evaluation on 5-7 and 30 day postoperatively based on Szilagyi classification.

\begin{tabular}{|c|c|c|c|c|c|c|}
\hline \multirow{2}{*}{ Szilagyi classification } & \multicolumn{3}{|l|}{ Total number } & \multicolumn{3}{|c|}{ 5-7 day postoperatively } \\
\hline & ciNPT-group $n=47$ & Control-group $n=53$ & p-value & ciNPT-group $n=47$ & Control-group $n=53$ & p-value \\
\hline Szilagyi grade I & $1(2.1 \%)$ & $11(20.7 \%)$ & 0.005 & $0(0 \%)$ & $7(13.2 \%)$ & 0.014 \\
\hline Szilagyi grade II & $5(10.6 \%)$ & $27(50.9 \%)$ & $<0.0005$ & $1(2.1 \%)$ & $19(35.8 \%)$ & $<0.0005$ \\
\hline Szilagyi grade III & $0(0 \%)$ & $1(1.9 \%)$ & 1 & $0(0 \%)$ & $0(0 \%)$ & - \\
\hline Total number & $6(12.7 \%)$ & $39(73.5 \%)$ & $<0.0005$ & $1(2.1 \%)$ & $26(49 \%)$ & $<0.0005$ \\
\hline
\end{tabular}


Table 3. Continued

\begin{tabular}{|c|c|c|c|c|c|c|}
\hline \multirow[b]{2}{*}{ Szilagyi classification } & \multicolumn{3}{|c|}{30 day postoperatively } & \multicolumn{3}{|c|}{ Revision surgery on 30 day postoperatively } \\
\hline & $\begin{array}{l}\text { ciNPT-group } \\
\mathrm{n}=47\end{array}$ & $\begin{array}{l}\text { Control-group } \\
\mathrm{n}=53\end{array}$ & p-value & $\begin{array}{l}\text { ciNPT-group } \\
\mathrm{n}=58\end{array}$ & $\begin{array}{l}\text { Control-group } \\
\mathrm{n}=71\end{array}$ & p-value \\
\hline Szilagyi grade I & $1(2.1 \%)$ & $4(7.5 \%)$ & 0.367 & $0(0 \%)$ & $2(2.8 \%)$ & 0.501 \\
\hline Szilagyi grade II & $4(8.5 \%)$ & $8(15.1 \%)$ & 0.368 & $1(1.7 \%)$ & $6(8.5 \%)$ & 0.128 \\
\hline Szilagyi grade III & $0(0 \%)$ & $1(1.9 \%)$ & 1 & $0(0 \%)$ & $2(2.8 \%)$ & 0.501 \\
\hline Total number & $5(10.6 \%)$ & $13(24.5 \%)$ & 0.116 & $1(1.7 \%)$ & $10(14.1 \%)$ & 0.022 \\
\hline
\end{tabular}

Table 4. Incidence of wound healing disturbances with reference to the revision surgery on 30 day postoperatively based on Szilagyi classification.

\begin{tabular}{llll}
\hline Szilagyi classification & ciNPT group & Control group & p-value \\
\hline Szilagyi grade I & $\mathrm{n}=47$ & $\mathrm{n}=53$ & \\
& $0(0 \%)$ & $0(0 \%)$ & - \\
Szilagyi grade II & $2(4.3 \%)$ & $8(15.1 \%)$ & 0.098 \\
Szilagyi grade III & $0(0 \%)$ & $1(1.9 \%)$ & 1 \\
Total number & $2(4.3 \%)$ & $9(17 \%)$ & 0.056 \\
\hline
\end{tabular}

Table 5. Types of wound complications within the three grades of Szilagyi classification.

\begin{tabular}{llll}
\hline & ciNPT group & Control group & p-value \\
\hline Superficial wound dehiscence & $1(2.1 \%)$ & $5(9.4 \%)$ & 0.210 \\
Skin necrosis & $1(2.1 \%)$ & $4(7.5 \%)$ & 0.367 \\
Deep wound dehiscence with fat necrosis & $0(0 \%)$ & $3(5.7 \%)$ & 0.245 \\
Hematoma & $2(4.2 \%)$ & $9(16.9 \%)$ & 0.056 \\
Seroma & $1(2.1 \%)$ & $5(9.4 \%)$ & 0.210 \\
Lymphatic fistula & $0(0 \%)$ & $6(11.3 \%)$ & 0.028 \\
Local infection & $1(2.1 \%)$ & $6(11.3 \%)$ & 0.117 \\
\hline
\end{tabular}

Table 6. Subgroup analysis with reference to the total number of groin incisions, wound evaluation on 5-7 and 30 day postoperatively based on Szilagyi classification.

\begin{tabular}{|c|c|c|c|c|c|c|}
\hline \multirow{2}{*}{ Subgroup Parameters } & \multicolumn{3}{|l|}{ Total number } & \multicolumn{3}{|c|}{ 5-7 day postoperatively } \\
\hline & ciNPT-group & Control-group & p-value & ciNPT-group & Control- group & p-value \\
\hline \multirow{2}{*}{ Age (>60 years) } & $\mathrm{n}=44$ & $\mathrm{n}=37$ & \multirow{2}{*}{$<0.0005$} & $\mathrm{n}=44$ & $\mathrm{n}=37$ & \multirow{2}{*}{$<0.0005$} \\
\hline & $5(11.4 \%)$ & $25(67.6 \%)$ & & $1(2.3 \%)$ & $16(43.2 \%)$ & \\
\hline \multirow{2}{*}{ Diabetes mellitus } & $\mathrm{n}=13$ & $\mathrm{n}=13$ & \multirow{2}{*}{$<0.0005$} & $\mathrm{n}=13$ & $\mathrm{n}=13$ & \multirow{2}{*}{0.005} \\
\hline & $0(0 \%)$ & $11(84.6 \%)$ & & $0(0 \%)$ & $7(53.8 \%)$ & \\
\hline \multirow{2}{*}{ Renal insufficiency } & $\mathrm{n}=19$ & $\mathrm{n}=12$ & \multirow{2}{*}{0.007} & $\mathrm{n}=19$ & $n=12$ & \multirow{2}{*}{0.005} \\
\hline & $1(5.3 \%)$ & $6(50 \%)$ & & $0(0 \%)$ & $5(41.7 \%)$ & \\
\hline \multirow{2}{*}{ Hypoproteinemia } & $\mathrm{n}=8$ & $\mathrm{n}=24$ & \multirow{2}{*}{0.003} & $\mathrm{n}=8$ & $\mathrm{n}=24$ & \multirow{2}{*}{0.029} \\
\hline & $1(12.5 \%)$ & $18(75 \%)$ & & $0(0 \%)$ & $11(45.8 \%)$ & \\
\hline \multirow{2}{*}{ Overweight } & $\mathrm{n}=39$ & $\mathrm{n}=35$ & \multirow{2}{*}{$<0.0005$} & $\mathrm{n}=39$ & $\mathrm{n}=35$ & \multirow{2}{*}{$<0.0005$} \\
\hline & $5(12.8 \%)$ & $24(68.6 \%)$ & & $1(2.6 \%)$ & $15(42.9 \%)$ & \\
\hline \multirow{2}{*}{ COPD } & $\mathrm{n}=10$ & $\mathrm{n}=5$ & \multirow{2}{*}{0.10} & $n=10$ & $\mathrm{n}=5$ & \multirow{2}{*}{0.004} \\
\hline & $1(10 \%)$ & $7(140 \%)$ & & $0(0 \%)$ & $6(120 \%)$ & \\
\hline \multirow{2}{*}{ Wound length (10 centimeter) } & $\mathrm{n}=19$ & $\mathrm{n}=24$ & \multirow{2}{*}{$<0.0005$} & $\mathrm{n}=19$ & $\mathrm{n}=24$ & \multirow{2}{*}{0.001} \\
\hline & $2(10.5 \%)$ & $22(91.7 \%)$ & & $1(5.3 \%)$ & $13(54.2 \%)$ & \\
\hline \multirow{2}{*}{ Hospital stay (18 days) } & $\mathrm{n}=16$ & $\mathrm{n}=12$ & \multirow{2}{*}{0.006} & $\mathrm{n}=16$ & $\mathrm{n}=12$ & \multirow{2}{*}{$<0.0005$} \\
\hline & $2(12.5 \%)$ & $15(125 \%)$ & & $0(0 \%)$ & $8(66.7 \%)$ & \\
\hline \multirow{2}{*}{$\begin{array}{l}\text { Operation time ( }>168 \\
\text { minutes) }\end{array}$} & $\mathrm{n}=20$ & $\mathrm{n}=21$ & \multirow{2}{*}{$<0.0005$} & $\mathrm{n}=20$ & $\mathrm{n}=21$ & \multirow{2}{*}{0.001} \\
\hline & $2(10 \%)$ & $15(74.1 \%)$ & & $0(0 \%)$ & $9(42.9 \%)$ & \\
\hline \multirow{2}{*}{ Previousinterventions } & $\mathrm{n}=2$ & $\mathrm{n}=4$ & \multirow{2}{*}{0.6} & $\mathrm{n}=2$ & $\mathrm{n}=4$ & \multirow{2}{*}{1} \\
\hline & $0(0 \%)$ & $5(125 \%)$ & & $0(0 \%)$ & $1(25 \%)$ & \\
\hline \multirow{2}{*}{ Perioperativeblood transfusion } & $\mathrm{n}=17$ & $\mathrm{n}=15$ & 0.003 & $\mathrm{n}=17$ & $\mathrm{n}=15$ & 0023 \\
\hline & $2(11.8 \%)$ & $10(66.7 \%)$ & 0.003 & $0(0 \%)$ & $8(53.3 \%)$ & 0.023 \\
\hline
\end{tabular}


Table 6. Continued

\begin{tabular}{|c|c|c|c|c|c|c|}
\hline \multirow{2}{*}{ Subgroup Parameters } & \multicolumn{3}{|c|}{30 day postoperatively } & \multicolumn{3}{|c|}{ Revision surgery on 30 day postoperatively } \\
\hline & ciNPT-group & Control-group & p-value & ciNPT-group & Control-group & p-value \\
\hline \multirow{2}{*}{ Age ( $>60$ years) } & $\mathrm{n}=44$ & $\mathrm{n}=37$ & \multirow[b]{2}{*}{0.075} & $\mathrm{n}=31$ & $\mathrm{n}=26$ & \multirow{2}{*}{0.029} \\
\hline & $4(9.1 \%)$ & $9(24.3 \%)$ & & $1(3.2 \%)$ & $6(23.1 \%)$ & \\
\hline \multirow{2}{*}{ Diabetes mellitus } & $\mathrm{n}=13$ & $\mathrm{n}=13$ & \multirow{2}{*}{0.096} & $\mathrm{n}=22$ & $\mathrm{n}=29$ & \multirow{2}{*}{0.061} \\
\hline & $0(0 \%)$ & $4(30.8 \%)$ & & $1(4.5 \%)$ & $7(24.1 \%)$ & \\
\hline \multirow{2}{*}{ Renal insufficiency } & $\mathrm{n}=19$ & $\mathrm{n}=12$ & \multirow{2}{*}{1} & $\mathrm{n}=27$ & $\mathrm{n}=30$ & \multirow{2}{*}{0.347} \\
\hline & $1(5,3 \%)$ & $1(8.3 \%)$ & & $1(3.7 \%)$ & $3(10 \%)$ & \\
\hline \multirow{2}{*}{ Hypoproteinemia } & $\mathrm{n}=8$ & $\mathrm{n}=24$ & \multirow{2}{*}{0.642} & $\mathrm{n}=13$ & $\mathrm{n}=22$ & \multirow{2}{*}{0.140} \\
\hline & $1(12.5 \%)$ & $7(29.2 \%)$ & & $0(0 \%)$ & $4(18.2 \%)$ & \\
\hline \multirow{2}{*}{ Overweight } & $\mathrm{n}=39$ & $\mathrm{n}=35$ & \multirow{2}{*}{0.125} & $\mathrm{n}=32$ & $\mathrm{n}=41$ & \multirow{2}{*}{0.167} \\
\hline & $4(10.3 \%)$ & $9(25.7 \%)$ & & $1(3.1 \%)$ & $5(12.2 \%)$ & \\
\hline \multirow{2}{*}{ COPD } & $\mathrm{n}=10$ & $\mathrm{n}=5$ & \multirow{2}{*}{1} & & $\mathrm{n}=8$ & \multirow{2}{*}{0.735} \\
\hline & $1(10 \%)$ & $1(20 \%)$ & & $1(11.1 \%)$ & $1(12.5 \%)$ & \\
\hline Wound length & $\mathrm{n}=19$ & $\mathrm{n}=24$ & \multirow{2}{*}{0.026} & $\mathrm{n}=25$ & $\mathrm{n}=49$ & \multirow{2}{*}{0.083} \\
\hline (10 centimeter) & $1(5.3 \%)$ & $9(37.5 \%)$ & & $1(4 \%)$ & $9(18.4 \%)$ & \\
\hline Hospital stay & $\mathrm{n}=16$ & $\mathrm{n}=12$ & \multirow{2}{*}{0.017} & $\mathrm{n}=37$ & $\mathrm{n}=48$ & \multirow{2}{*}{0.012} \\
\hline (18 days) & $2(12.5 \%)$ & $7(58 \%)$ & & $1(2.7 \%)$ & $10(20.8 \%)$ & \\
\hline Operation time & $\mathrm{n}=20$ & $\mathrm{n}=21$ & \multirow{2}{*}{0.238} & $\mathrm{n}=21$ & $\mathrm{n}=28$ & \multirow{2}{*}{0.062} \\
\hline (> 168 minutes) & $2(10 \%)$ & $6(28.6 \%)$ & & $1(4.7 \%)$ & $7(25 \%)$ & \\
\hline Previous & $\mathrm{n}=2$ & $\mathrm{n}=4$ & \multirow{2}{*}{0.067} & $\mathrm{n}=9$ & $\mathrm{n}=18$ & \multirow{2}{*}{0.279} \\
\hline interventions & $0(0 \%)$ & $4(100 \%)$ & & $0(0 \%)$ & $3(16.7 \%)$ & \\
\hline Perioperative & $\mathrm{n}=17$ & $\mathrm{n}=15$ & \multirow{2}{*}{1} & $\mathrm{n}=9$ & $\mathrm{n}=13$ & \multirow{2}{*}{0.642} \\
\hline blood transfusion & $2(11.8 \%)$ & $2(13.3 \%)$ & & $1(11.1 \%)$ & $2(15.4 \%)$ & \\
\hline
\end{tabular}

Table 7. Subgroup analysis with reference to revision surgeries on 30 day postoperatively based on Szilagyi classification.

\begin{tabular}{llll}
\hline Subgroup postoperatively parameters & ciNPT group & Control group & p-value \\
\hline Age ( $>60$ years) & $\mathrm{n}=44$ & $\mathrm{n}=37$ & 0.133 \\
& $2(4.5 \%)$ & $6(16.2 \%)$ & 0.096 \\
Diabetes mellitus & $\mathrm{n}=13$ & $\mathrm{n}=13$ & 1 \\
Renal insufficiency & $0(0 \%)$ & $4(30.8 \%)$ & $\mathrm{n}=12$ \\
& $\mathrm{n}=19$ & $0(0 \%)$ & 0.646 \\
Hypoproteinemia & $1(5.3 \%)$ & $\mathrm{n}=24$ & \\
& $\mathrm{n}=8$ & $6(25 \%)$ & 0.139 \\
Overweight & $1(12.5 \%)$ & $\mathrm{n}=35$ & 1 \\
& $\mathrm{n}=39$ & $6(17.1 \%)$ & $\mathrm{n}=5$ \\
COPD & $2(5.1 \%)$ & $0(0 \%)$ & 0.027 \\
Wound length (10 centimeter) & $\mathrm{n}=10$ & $\mathrm{n}=24$ & 0.017 \\
Hospital stay (18 days) & $0(0 \%)$ & $0(25 \%)$ & $\mathrm{n}=12$ \\
Operation time (>142 minutes) & $\mathrm{n}=19$ & $7(58.3 \%)$ & $\mathrm{n}=21$ \\
& $0(0 \%)$ & $\mathrm{n}=16$ & 0.343 \\
Previous interventions & $2(12.5 \%)$ & $\mathrm{n}=20$ & 1 \\
Perioperative blood transfusion & $1(5 \%)$ & $1(25 \%)$ & $\mathrm{n}=15$ \\
\hline
\end{tabular}

\section{Discussion}

The potential of ciNPT to prevent or reduce postoperative WHCs in various surgical disciplines has already been demonstrated in many publications over the past decade [510, 16-24, 27-29]. In contrast, study data concerning postoperative WHCs in the groin after vascular surgery are less common [5-10, 16, 25-29]. In all of these studies PREVENA $^{\mathrm{TM}}$ and PICO $^{\mathrm{TM}}$ were the leading ciNPT systems applied. Matatov et al. first reported a reduction of infections in postoperative incision wounds in the groin $(p=0.011)$ when PREVENA $^{\mathrm{TM}}$ was used instead of adhesive and absorbent dressings [5]. In subsequent years various randomized clinical studies confirmed the effect of applying ciNPT to postoperative incision groin wounds $[6-8,16,27,29]$. Pleger et al. demonstrated a reduction in WHCs $(\mathrm{p}<0.0005)$ and revision surgeries $(\mathrm{p}=0.022)$ in postoperative incision groin wounds treated by PREVENA ${ }^{\mathrm{TM}}$ in comparison with a conventional wound dressing [6]. Gombert et al. showed a reduction in SSI prevalences in groin incisions after vascular surgery in which PREVENA ${ }^{\mathrm{TM}}$ was applied instead of a conventional wound dressing $(p=0.015)$ [7]. Furthermore, Hasselmann et al. reported lower number of SSIs in patients with unilateral and bilateral groin incisions after vascular surgery when treated with $\mathrm{PICO}^{\mathrm{TM}}(\mathrm{p}=0.02)$ [16], and Kwon 
et al. published data showing a reduction in wound complications $(p<0.001)$ and reoperation $(p<0.05)$ using PREVENATM $^{\mathrm{TM}}$ versus standard wound dressing [28]. These results have been supported by some meta-analyses $[9,10$, 29]. Gombert et al. reported a reduced incidence of SSIs in groin incisions after analyzing six RCTs comprising a total of 733 patients treated with PREVENA ${ }^{\mathrm{TM}}$ or standard dressing (odds ratio [OR] 3.06, 95\% confidence intervals [CI] [2.05 4.58]; $\mathrm{p}<0.05)$. Moreover, Svensson et al. analysis of seven RCTs of vascular surgery patients confirmed a reduction in the incidence of SSIs in the groin after using PREVENA ${ }^{\text {TM }}$ and $\mathrm{PICO}^{\mathrm{TM}}$ versus standard dressing in vascular surgery patients (OR 0.35, 95\% CI [0.24 - 0.50], p<0.001) [10], and Singh et al. found a significant effect for PREVENA ${ }^{\text {TM }}$ in preventing SSIs $(\mathrm{p}<0.0001)$ (OR 3.17, 95\% CI [2.17 - 4.65], $\mathrm{p}<0.001)$ after analyzing 17 articles concerning the effect of PREVENA $^{\mathrm{TM}}$ and PICO $^{\mathrm{TM}}$ versus standard dressing [29]. Despite these positive results, it must be mentioned that there are also data from studies of PREVENA ${ }^{\mathrm{TM}}$ that did not show a significant effect of the treatment. For example, in a study by Sabat et al. reduction of wound dehiscence $(p=0.14)$ or wound infection $(p=0.09)$ was not significant [8]; overall wound infection rates in a study by Engelhardt et al. were not significant $(\mathrm{p}=0.055)$ [26], and there was no effect on wound healing disorders $(\mathrm{p}=0.552)$ or SSIs $(\mathrm{p}=0.458)$ as reported by Koetje et al. [25]. Taking the above-mentioned studies into account, almost all data refer to the healing of primary groin incision. Considering the fact that after initial vascular surgery, patients often need a second surgical procedure, wound healing in the groin after revision surgeries is a highly relevant aspect. Unfortunately, RCT data randomized clinical data on the preventive effect of ciNPT on revision WHCs in the groin are very rare. In the previously mentioned RCT by Gombert et al., 85 of 204 patients (ciNPT n=46; control group $n=39$ ) had a previous incision in the groin. Among these patients SSIs were less frequent in the ciNPT group than in the control group $(n=5[10.8 \%]$ vs. $n=13$ [33.3\%], respectively); based on this result an effect of ciNPT (PREVENA $\left.{ }^{\mathrm{TM}}\right)$ was clear $(p=0.016)$ [7]. Further data considering previous groin incision wounds were reported by Lee et al.: in 30 of 102 high-risk patients with a previous cutdown in the groin, the application of ciNPT (PREVENA $\left.^{\mathrm{TM}}\right)(\mathrm{n}=13)$ or standard wound dressing $(\mathrm{n}=17)$ after redo vascular surgery showed no difference in SSI frequency between the two treatment methods $(p=0.24)$ [27]. With these published results as a backdrop, in our study including 94 patients with 100 groin incisions, WHCs were observed only in $6(12.8 \%)$ patients in the ciNPT group, whereas $39(73.6 \%)$ patients from the control group experienced various postoperative WHCs (Tables 3 and 5). The overall incidence of postoperative WHCs clearly showed that ciNPT (PREVENA ${ }^{\mathrm{TM}}$ ) was more favorable than the conventional adhesive dressing $(p<0.0005$; Table 3$)$. Our data confirm the results of Gombert et al. by proving a significant effect of ciNPT (PREVENA ${ }^{\mathrm{TM}}$ ) in reducing WHCs in groin incision after revision vascular surgery. With regard to WHCs needing for a surgical revision, our data show $12.8 \%$ fewer revision surgeries in the PREVENA ${ }^{\mathrm{TM}}$ group at 30 days postoperatively compared with to the control group ( $4.3 \%$ vs. $17 \%$, respectively), although this difference did not reach significance $(\mathrm{p}=0.056)$. We observed a markedly better result on postoperative day 5-7 $(\mathrm{p}<0.0005)$, than on day $30(p=0.116)$ (Table 3$)$. The explanation for this reduced effect of ciNPT between the two evaluations periods may be that the duration of application was too short. This aspect is supported by the increase in the frequency of WHCs in the ciNPT group, resulting in a difference of $8.5 \%(2.1 \%$ vs. $10.6 \%$ ). Similar observations were made by Engelhardt et al. that showed a difference in the frequency of SSIs between postoperative days 5 and 42 of $8 \%$ [26], and Pleger et al. reported a difference in WHC occurrence between postoperative days $5-7$ and 30 of $8.6 \%$ [6]. Although these results relate to primary incision wounds in the groin, the similarity to our present study data $(8.5 \%$ vs. $8 \%$ and $8.6 \%)$ is consistent with the loss of effect of ciNPT in both types of incision wounds. Since the published RCTs do not report detailed information for individual evaluation days, a comparison to our observation is hardly possible. Due to this lack of data, further studies need to clarify the question regarding the most effective period of application of ciNPT in primary and revision incision wounds in the groin. Our subgroup analysis of the wound healing risk factors and perioperative risk factors significantly fewer WHCs in patients treated with ciNPT for all risk factors investigated except COPD and previous interventions (Table 6). In patients with wound revision, a significant effect was revealed only for the risk factors wound length $(p=0.027)$ and hospital stay $(\mathrm{p}=0.017)$ (Table 7$)$. These results demonstrate a high susceptibility of these decisive wound healing risk factors for ciNPT and reveal at the same time the potential benefits of this treatment in patients with exactly this profile of risk factors. Pleger et al. detected a benefit for patients with similar risk factors, showing a high relevance for targeted use of the ciNPT [6]. In addition, Gombert et al. identified $B M I>25 \mathrm{~kg} / \mathrm{m}^{2} \quad(\mathrm{p}<0.001)$ and peripheral artery disease stage $\geq 3(p<0.001)$ as risk factors that lead to a higher rate of SSIs [7], and Lee et al. revealed a shorter duration of hospital stay $(\mathrm{p}=0.02)$ in ciNPT patients, emphasizing the importance of ciNPT with regard to these risk factors [27]. A further notable aspect of our study is the fewer lymphatic fistulae in the ciNPT group (ciNPT $n=0$ vs. control group $\mathrm{n}=6 ; \mathrm{p}=0.028$ ). Similarly, Kwon et al. also observed a reduction in lymph leakage using the ciNPT ( ciNPT $n=0$ vs. control group $n=2$ ) [28], and Pleger et al. reported a decreased number of lymphatic fistulae (ciNPT $n=1$ vs. control group $n=3$ ) [6]. These results highlight a promising effect of ciNPT in preventing lymphatic leakages, which can lead to frequent revision surgeries resulting in longer hospital stay and higher treatment costs. Ragarding this latter consideration, an analysis by Kwon et al. of the financial impact of ciNPT revealed a variable hospital cost savings of more than $\$ 6000$ per patient despite a lack of statistical significance $(p=0.11)$ [28]. Unfortunately, cost analyses with regard to the use of ciNPT for incision wounds 
in the groin are scarce; thus, a conclusive statement concerning an economic advantage cannot be made. Despite the positive effect of ciNPT observed in our study it has to be considered that postoperative wound healing in the groin after vascular surgery is not only dependent on ciNPT and its period of application. In addition, other factors including a redo cutdown in the groin, wound contamination during wound dressing changes, body hygiene, too early postoperative movement in the groin, and surgical preparation technique can negatively affect the wound healing process in the groin. Although our results show a significant reduction in the frequency of WHCs and thereby suggest that present ciNPT is a promising therapeutic option in the treatment of incision wounds in the groin after revision vascular surgery, this therapy cannot yet be generalized to standard of care, and further studies on revision wounds are required to substantiate our data.

\section{Limitations}

The evaluation of the incision wounds that was carried out by the investigators of the study should be mentioned as a protentional limitation. The lack of blinding in the evaluation procedure could have led to assessment bias, which could have been avoided by a double-blinded study design.

In addition, as the evaluation time period as restricted to 30 days the observation of probable WHCs during subsequent days was not possible. A longer observation period could resolve this uncertainty and might show a longterm effect of ciNPT.

\section{Conclusion}

With the limited study data regarding postoperative WHCs in groin incision wounds after revision vascular surgery as a backdrop, our results show a significant reduction in the overall incidence of postoperative WHCs and underline the ciNPT as a promising therapeutic approach to minimize the frequency of postoperative WHCs and the need for revision surgeries.

In addition, the subgroup analysis revealed a significant effect of ciNPT on almost all risk factors examined. Assessment of specific risk factors will allow individualized indication for the application of ciNPT in patients at risk and thus prevent arbitrary use of this therapy.

Due to an increase in the costs of treating postoperative groin WHCs, an additional cost analysis to assess a potential economic advantage of ciNPT versus conventional wound dressing may support the application of ciNPT and make use of its preventive effect. A cost analysis of our results is planned in a future investigation.

\section{Conflict of Interests}

The authors declare that they have no competing interests.

\section{References}

[1] National Healthcare Safety Network (HSN) 2019 Surgical Site Infection (SSI) Protocol:

https://www.cdc.gov/nhsn/pdfs/pscmanual/9pscssicurrent.pdf.

[2] Magill, SS, Edwards JR, Bamberg W, Beldavs ZG, Dumyati G, et al. Multistate point-prevalence survey of health careassociated infections. New England Journal of Medicine. 2014; 370 (13): 1198-1208.

[3] de Lissovoy G, Fraeman K, Hutchins V, Murphy D, Song D, et al. Surgical site infection: incidence and impact on hospital utilization and treatment costs. Am J Infect Control. 2009; 37 (5): 387-97.

[4] Condon, R. E., Schulte WJ, Malangoni MA, AndersonTeschendorf MJ. Effectiveness of a surgical wound surveillance program. Archives of Surgery. 1983; 118 (3): 303-7.

[5] Matatov T, Reddy KN, Doucet LD, Zhao CX, Zhang WW. Experience with a new negative pressure incision management system in prevention of groin wound infection in vascular surgery patients. J Vasc Surg. 2013; 57 (3): 791-5.

[6] Pleger SP, Nink N, Elzien M, Kunold A, Koshty A, et al. Reduction of groin wound complications in vascular surgery patients using closed incision negative pressure therapy (ciNPT): a prospective, randomised, single-institution study. Int Wound J. 2018; 15 (1): 75-83.

[7] Gombert A, Babilon M, Barbati ME, Keszei A, von Trotha KT, et al. Closed Incision Negative Pressure Therapy Reduces Surgical Site Infections in Vascular Surgery: A Prospective Randomised Trial (AIMS Trial). Eur J Vasc Endovasc Surg. 2018; 56 (3): 442-8.

[8] Sabat J, Tyagi S, Srouji A, Pechman D, Gupta AM, et al. Prophylactic negative pressure therapy for femoral incision in vascular surgery: preliminary results of a prospective randomised trial. Journal of Vascular Surgery. 2016; 63: 94S.

[9] Gombert A, Dillavou E, D’Agostino R, Griffin L, Robertson $\mathrm{JM}$, et al. A systematic review and meta-analysis of randomized controlled trials for the reduction of surgical site infection in closed incision management versus standard of care dressings over closed vascular groin incisions. Vascular. 2020; 28 (3): 274-84.

[10] Svensson-Björk R, Zarrouk M, Asciutto G, Hasselmann J, Acosta $\mathrm{S}$, et al. Meta-analysis of negative pressure wound therapy of closed groin incisions in arterial surgery, BJS. 2019; 106 (4): 310-8.

[11] Dosluoglu HH, Loghmanee C, Lall P, Cherr GS, Harris LM, et al. Management of early ( $<30$ day) vascular groin infections using vacuum-assisted closure alone without muscle flap coverage in a consecutive patient series. J Vasc Surg. 2010; 51: 1160-6.

[12] Stannard JP, Robinson JT, Anderson ER, McGwin G, Volgas $\mathrm{DA}$, et al. Negative pressure wound therapy to treat hematomas and surgical incisions following high-energy trauma. J Trauma. 2006; 60: 1301-6.

[13] Gustafsson R, Johnsson P, Algotsson L, Blomquist S, Ingemansson R. Vacuum-assisted closure therapy guided by C-reactive protein level in patients with deep sternal wound infection. J Thorac Cardiovasc Surg. 2002; 123: 895-900. 
[14] Wongworawat MD, Schnall SB, Holtom PD, Moon C, Schiller F. Negative pressure dressings as an alternative technique for the treatment of infected wounds. Clin Orthop. 2003; 414: 45-8.

[15] DeFranzo AJ, Argenta LC, Marks MW, Molnar JA, David LR, et al. The use of vacuum-assisted closure therapy for the treatment of lower-extremity wounds with exposed bone. Plast Reconstr Surg. 2001; 108: 1184-91.

[16] Hasselmann J, Björk J, Svensson-Björk R, Acosta S. Inguinal Vascular Surgical Wound Protection by Incisional Negative Pressure Wound Therapy: A Randomized Controlled TrialINVIPS Trial. Ann Surg. 2020; 271 (1): 48-53.

[17] Grauhan O, Navasardyan A, Tutkun B, Hennig F, Müller P, et al. Effect of surgical incision management on wound infections in a poststernotomy patient population. Int Wound $\mathrm{J}$. 2014; 11 Suppl 1: 6-9.

[18] Pachowsky M, Gusinde J, Klein A, Lehrl S, Schulz-Drost S. Negative pressure wound therapy to prevent seromas and treat surgical incisions after total hip arthroplasty. Int Orthop. 2012; 36 (4): 719-22.

[19] Conde-Green A, Chung TL, Holton LH, Hui-Chou HG, Zhu Y, et al. Incisional Negative-Pressure Wound Therapy versus conventional dressings following abdominal wall reconstruction. A comparative study. Ann Plast Surg. 2013; 71 (4): 394-7.

[20] Stannard JP, Volgas DA, McGwin G, Stewart RL, Obremskey $\mathrm{W}$, et al. Incisional negative pressure wound therapy after high-risk lower extremity fractures. J Orthop Trauma. 2012; 26 (1): $37-42$

[21] Anglim B, Oconnor H, Daly S. PrevenaTM negative pressure wound therapy applied to closed Pfannenstiel incisions at time of caesarean section in patients deemed at high risk for wound infection. J Obstet Gynaecol. 2015; 35 (3): 255-8.

[22] Galiano RD, Hudson D, Shin J, van der Hulst R, Tanaydin V, et al. Incisional Negative Pressure Wound Therapy for
Prevention of Wound Healing Complications Following Reduction Mammaplasty. Plast Reconstr Surg Glob Open. 2018; 6 (1): e1560.

[23] Fowler AL, Barry MK. Closed incision negative pressure therapy for laparotomy wounds: A review. Clin Surg. 2018; 3: 2123.

[24] Horch RE. Incisional negative pressure wound therapy for high-risk wounds. J Wound Care 2015; 24: 21-8.

[25] Koetje JH, Ottink KD, Feenstra I, Fritschy WM. Negative Pressure Incision Management System in the Prevention of Groin Wound Infection in Vascular Surgery Patients. Surgery Research and Practice. 2015; 2015: 303560.

[26] Engelhardt M, Rashad NA, Wily C, Müller C, Bauer C, et al. Closed-incision negative pressure therapy to reduce groin wound infections in vascular surgery: a randomised controlled trial. Int Wound J. 2018; 15 (3): 327-32.

[27] Lee K, Murphy PB, Ingves MV, Duncan A, DeRose G, et al. Randomized clinical trial of negative pressure wound therapy for high-risk groin wounds in lower extremity revascularization. J Vasc Surg. 2017; 66 (6): 1814-9.

[28] Kwon J, Staley C, McCullough M, Goss S, Arosemena M, et al. A randomized clinical trial evaluating negative pressure therapy to decrease vascular groin incision complications. J Vasc Surg. 2018; 68 (6): 1744-52.

[29] Singh DP, Gabriel A, Silverman RP, Griffin LP, D'Agostino McGowan L, et al. Meta-analysis Comparing Outcomes of Two Different Negative Pressure Therapy Systems in Closed Incision Management. Plast Reconstr Surg Glob Open. 2019; 21; 7 (6): e2259.

[30] Szilagyi DE, Smith RF, Elliott JP, Vrandecic MP. Infection in arterial reconstruction with synthetic grafts. Ann Surg. 1972; 176: 321-33. 